\title{
Characterization of sound scattering using near-field pressure and particle velocity measurements
}

Richard, Antoine; Fernández Comesaña, Daniel; Brunskog, Jonas; Jeong, Cheol Ho; Fernandez-Grande, Efren

Published in:

Journal of the Acoustical Society of America

Link to article, DOI:

$10.1121 / 1.5126942$

Publication date:

2019

Document Version

Publisher's PDF, also known as Version of record

Link back to DTU Orbit

Citation (APA):

Richard, A., Fernández Comesaña, D., Brunskog, J., Jeong, C. H., \& Fernandez-Grande, E. (2019).

Characterization of sound scattering using near-field pressure and particle velocity measurements. Journal of the Acoustical Society of America, 146(4), 2404-2414. https://doi.org/10.1121/1.5126942

\section{General rights}

Copyright and moral rights for the publications made accessible in the public portal are retained by the authors and/or other copyright owners and it is a condition of accessing publications that users recognise and abide by the legal requirements associated with these rights.

- Users may download and print one copy of any publication from the public portal for the purpose of private study or research.

- You may not further distribute the material or use it for any profit-making activity or commercial gain

- You may freely distribute the URL identifying the publication in the public portal 


\title{
Characterization of sound scattering using near-field pressure and particle velocity measurements ${ }^{\text {a) }}$
}

\author{
Antoine Richard, ${ }^{1}$ Daniel Fernández Comesaña, ${ }^{2}$ Jonas Brunskog, ${ }^{1}$ Cheol-Ho Jeong, ${ }^{1}$ \\ and Efren Fernandez-Grande ${ }^{1, b)}$ \\ ${ }^{1}$ Acoustic Technology, Department of Electrical Engineering, Technical University of Denmark (DTU), \\ Building 352, DK-2800 Kongens Lyngby, Denmark \\ ${ }^{2}$ Microflown Technologies, Tivolilaan 205, Arnhem, The Netherlands
}

(Received 28 February 2019; revised 30 August 2019; accepted 6 September 2019; published online 14 October 2019)

\begin{abstract}
The acoustic properties of surfaces are commonly evaluated using samples of finite size, which generate edge diffraction effects that are often disregarded. This study makes use of sound scattering theory to characterize such finite samples. In a given sound field, the samples can be described by a unique complex directivity function called the far-field pattern. Numerical results show that the far-field pattern contains extensive information on the tested samples, including sound absorption and surface scattering, as well as scattering due to finiteness. In this paper, a method is introduced to estimate the far-field pattern of a finite sample. The method relies on measurements of the sound pressure and acoustic particle velocity in the near-field of the sample, and it makes use of the Helmholtz integral equation. The proposed technique is examined in an anechoic room where the sound field near the test sample is scanned with a three-dimensional sound intensity probe. The estimated far-field pattern is compared with numerical predictions up to $1 \mathrm{kHz}$.
\end{abstract}

(C) 2019 Acoustical Society of America. https://doi.org/10.1121/1.5126942

[MV]

Pages: $2404-2414$

\section{INTRODUCTION}

The characterization of the acoustical properties of surfaces has been the subject of extensive research as they are an essential input to room acoustics models. ${ }^{1}$ Surfaces are typically described in terms of sound absorption (energy dissipation in the material $)^{2}$ and scattering (energy dispersion due to surface roughness). ${ }^{3}$ Standards have also been proposed to measure both surface absorption ${ }^{4,5}$ and surface scattering. ${ }^{6,7}$ Current measures include the absorption coefficient, ${ }^{8}$ scattering coefficient, ${ }^{9}$ and diffusion coefficient. ${ }^{10}$ The definitions of these coefficients are based on the assumption that the surfaces are infinitely large so that they are suitable for high-frequency models such as geometrical acoustics. ${ }^{1}$

However, in actual rooms and measurement setups, surfaces are inevitably of finite size so the behavior of the reflected sound field deviates from the infinite panel theory, especially at low frequencies. ${ }^{11}$ This is also evidenced when modeling scattering by a rectangular panel, which does not lead to a specular reflection. ${ }^{12}$ These discrepancies lead to errors in experimental estimations, e.g., for the absorption coefficient. ${ }^{13}$ Furthermore, some of the existing metrics present conceptual challenges related to finiteness. In the case of the scattering coefficient, which quantifies the amount of non-specular energy in the reflection, additional assumptions are needed to define the specular reflection for finite samples. For instance, the specular reflection can be modeled as the part of the reflection that is coherent by rotation. ${ }^{6,9}$

\footnotetext{
a) Portions of this work were presented in "Characterization of acoustic scattering from objects via near-field measurements," Proceedings of Euronoise 2018, May 2018, Crete, Greece.

${ }^{b)}$ Electronic mail: efg@elektro.dtu.dk
}

Finally, reflection by a finite surface is a complex process, which cannot be entirely described by a single figure. That is why more complete representations of the reflection, including spatial information, have also been proposed, such as the directional scattering coefficient (DISC). ${ }^{14,15}$

In order to conform to the theory, methods have been suggested to exclude, or at least minimize, finite-size effects, both for the measurement of absorption and surface scattering. Using large enough samples may be the easiest solution, ${ }^{16}$ although too large samples can become impractical in experimental setups. Thomasson suggested to apply a correction to the measured absorption coefficient at random incidence to account for edge effects. ${ }^{17}$ In terms of surface scattering, it has been recommended to use circular samples in the standardized rotation method so that edge effects can be considered invariant and, thus, coherent by rotation. ${ }^{18}$ Note that the finite size effect is, then, still included in the estimated specular energy. Alternatively, it has also been proposed to include the effect of sample size in the metric. For instance, the finite size effect can be included in an absorption coefficient by modeling it as a radiation impedance. ${ }^{19}$ Such an approach can be more suitable for studying sound scattering, which is, in fact, both due to surface roughness and the sample's shape.

More generally, the reflection of sound by a given finite sample can be studied as a general object scattering problem, i.e., by comparing the sound field with and without the sample. In that sense, scattering can be understood as the combination of all disturbances on the sound field caused by the sample. This includes traditional rough surface scattering, but also absorption and effects due to the size and shape of the sample. This process benefits from a strong 
mathematical background. ${ }^{20}$ In particular, an object can be characterized by a far-field directivity function, which is uniquely defined. Therefore, it is possible to use such a function to describe acoustic samples of finite size such as absorbers and diffusers. We refer to this function as far-field pattern, following Ref. 20, but it appears in the literature under many other names, for instance, as directivity factor ${ }^{21}$ or scattering amplitude. ${ }^{22}$ Note that directivity measures exist to characterize surfaces, such as the DISC $^{14}$ and the bidirectional reflectance distribution function (BRDF), ${ }^{23}$ but a fundamental difference is that the far-field pattern explicitly includes finite size effects. Moreover, using a far-field measure aligns with the architectural acoustics literature, which recommends to study surface scattering in the farfield, both in terms of measurements ${ }^{14,15}$ and modeling. ${ }^{24,25}$

The far-field pattern requires large receiver distances to be measured directly. ${ }^{10}$ However, it is possible to make use of near-field measurements in order to extrapolate far-field information. This has been achieved for assessing surface scattering using planar arrays ${ }^{15}$ or spherical arrays. ${ }^{26} \mathrm{We}$ propose to extend the principle where the far-field pattern is directly inferred from near-field pressure and particle velocity measurements on an arbitrary domain. Such data can be measured with the advent of particle velocity sensors. ${ }^{27}$

This study aims at examining the properties of the farfield pattern for finite acoustic samples, and especially at investigating its relation to traditional architectural acoustics concepts such as absorption and surface scattering. The samples are studied under plane wave incidence in the free-field, so that the far-field pattern only depends on frequency and an incidence angle. The proposed measurement principle, i.e., predicting far-field properties from near-field data, is validated experimentally. Section II presents object scattering concepts and the proposed near-field measurement methodology. Section III examines the properties of the far-field pattern through numerical examples. The measurement method is validated in Sec. IV. Finally, the approach is discussed in Sec. V.

\section{METHODOLOGY}

This section introduces useful object scattering concepts for characterizing arbitrary acoustic samples, and presents a near-field measurement method to estimate the far-field pattern based on the Helmholtz integral equation (HIE).

\section{A. Object scattering and far-field pattern}

The formalization of object scattering appears in various scientific fields. In this paper, we refer to Ref. 20, which presents inverse scattering problems in acoustics and electromagnetics.

We consider a homogeneous medium characterized by its speed of sound $c$ and density $\rho$. Let a point in the medium be located at a distance $r$ from an arbitrary origin $O$ in the direction of a unitary vector $\hat{\mathbf{r}}$. The point can be represented by the vector $\mathbf{r}=r \hat{\mathbf{r}}$ (see Fig. 1). The direction indicated by $\hat{\mathbf{r}}$ can also be specified as a set of a polar angle and an azimuth angle in spherical coordinates. The undisturbed pressure at the location $\mathbf{r}$ is noted $p_{0}(\mathbf{r})$. The introduction of an

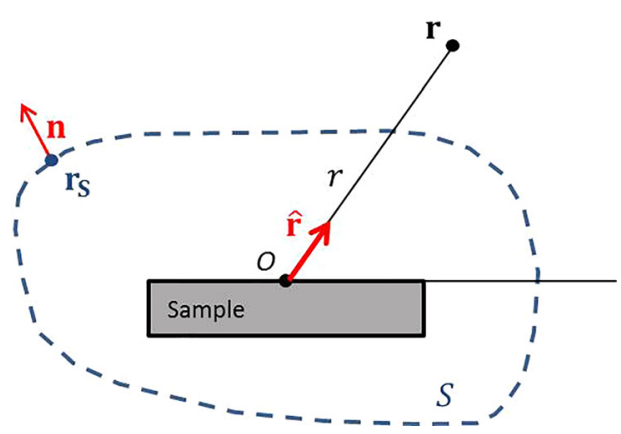

FIG. 1. (Color online) Sketch of the setup on which the Helmholtz integral equation is applied. The scattered field is calculated at the position $\mathbf{r}$ and the integral is calculated on the surface $S$.

object in the medium modifies the sound field, and the resulting pressure at $\mathbf{r}$ is called the total pressure $p_{t}(\mathbf{r})$. The scattered pressure $p_{s}(\mathbf{r})$ is defined as

$$
p_{s}(\mathbf{r})=p_{t}(\mathbf{r})-p_{0}(\mathbf{r}) .
$$

Note that while $p_{0}(\mathbf{r})$ and $p_{t}(\mathbf{r})$ are physical quantities that can potentially be measured, $p_{s}(\mathbf{r})$ is a mathematical construction.

The scattered field fulfills Sommerfeld's radiation condition, which makes it possible to express it in the far-field as

$$
p_{s}(\mathbf{r}) \underset{r \rightarrow+\infty}{=} \frac{e^{-j k r}}{r}\left(f_{\infty}(\hat{\mathbf{r}})+O\left(\frac{1}{r}\right)\right),
$$

where $k$ is the wavenumber. Equation (2) shows that the radial and angular dependence of $p_{s}$ can be separated in the far-field. The radial part follows a spherical decay, whereas the angular part $f_{\infty}(\hat{\mathbf{r}})$ is a complex directivity function called the far-field pattern. ${ }^{20}$ For a given undisturbed sound field $p_{0}$, the far-field pattern is uniquely determined by the object, and is therefore directly linked to the scattering properties of the object.

\section{B. HIE and application}

We study scattering of a plane wave by a given acoustic sample. We focus on flat samples that have an absorptive or diffusive upper surface, but the method can be applied to any other object with any shape and boundary conditions.

Let $S$ be an arbitrary surface enclosing the sample, as shown in Fig. 1. $S$ is assumed to be sufficiently close to the sample so that it does not contain any sound source. The HIE relates the scattered pressure at a given position $\mathbf{r}$ to the scattered sound field on $S$. Outside of the surface, the scattered pressure is given by ${ }^{20}$

$$
p_{s}(\mathbf{r})=\iint_{S}\left(p_{s}\left(\mathbf{r}_{\mathbf{S}}\right) \frac{\partial G\left(\mathbf{r}, \mathbf{r}_{\mathbf{S}}\right)}{\partial n}-\frac{\partial p_{s}\left(\mathbf{r}_{\mathbf{S}}\right)}{\partial n} G\left(\mathbf{r}, \mathbf{r}_{\mathbf{S}}\right)\right) d S\left(\mathbf{r}_{\mathbf{S}}\right)
$$

where $\mathbf{r}_{\mathbf{S}}$ is a point on the surface $S, \partial / \partial n$ is the normal derivative with respect to $S$, and $G$ is a Green's function, solution to the Helmholtz equation, which is assumed to be known analytically. A significant property of Eq. (3) is that 
if no source is enclosed by $S$, then it is also valid when $p_{s}$ is replaced by the total pressure $p_{t}$ in the integral.

In the right-hand side of Eq. (3), $\mathbf{r}$ only appears in $G\left(\mathbf{r}, \mathbf{r}_{\mathbf{S}}\right)$. Furthermore, assuming that $G$ is a radiating solution, it has the same form as Eq. (2) in the far-field,

$$
G\left(\mathbf{r}, \mathbf{r}_{\mathbf{S}}\right) \underset{r \rightarrow+\infty}{=} \frac{e^{-j k r}}{r}\left(G_{\infty}\left(\hat{\mathbf{r}}, \mathbf{r}_{\mathbf{S}}\right)+O\left(\frac{1}{r}\right)\right) .
$$

The term $G_{\infty}$ can be calculated analytically. For instance, if we consider the free-field Green's function due to a point source at the position $\mathbf{r}_{S}$

$$
G^{\prime}\left(\mathbf{r}, \mathbf{r}_{S}\right)=\frac{e^{-j k|| \mathbf{r}-\mathbf{r}_{S} \|}}{4 \pi\left\|\mathbf{r}-\mathbf{r}_{S}\right\|}
$$

the corresponding far-field function $G_{\infty}^{\prime}$ in the direction given by $\hat{\mathbf{r}}$ is ${ }^{20}$

$$
G_{\infty}^{\prime}\left(\hat{\mathbf{r}}, \mathbf{r}_{S}\right)=\frac{e^{j k \hat{\mathbf{r}} \cdot \mathbf{r}_{S}}}{4 \pi}
$$

When $r \rightarrow+\infty$, Eq. (4) can be inserted into Eq. (3). The term $e^{-j k r} / r$ is factorized, and by identification, one obtains

$$
\begin{aligned}
f_{\infty}(\hat{\mathbf{r}})= & \iint_{S}\left(p_{s}\left(\mathbf{r}_{\mathbf{S}}\right) \frac{\partial G_{\infty}\left(\hat{\mathbf{r}}, \mathbf{r}_{\mathbf{S}}\right)}{\partial n}\right. \\
& \left.-\frac{\partial p_{s}\left(\mathbf{r}_{\mathbf{S}}\right)}{\partial n} G_{\infty}\left(\hat{\mathbf{r}}, \mathbf{r}_{\mathbf{S}}\right)\right) d S\left(\mathbf{r}_{\mathbf{S}}\right) .
\end{aligned}
$$

Equation (7) relates the far-field pattern of the sample to the sound field on the surface $S$. $\partial p_{s} / \partial n$ is proportional to the normal particle velocity $u_{s, n}$ through Euler's equation of motion, so Eq. (7) can also be expressed as a function of the pressure and the normal particle velocity on $S$,

$$
\begin{aligned}
f_{\infty}(\hat{\mathbf{r}})= & \iint_{S}\left(p_{s}\left(\mathbf{r}_{\mathbf{S}}\right) \frac{\partial G_{\infty}\left(\hat{\mathbf{r}}, \mathbf{r}_{\mathbf{S}}\right)}{\partial n}\right. \\
& \left.+j \rho c k u_{s, n}\left(\mathbf{r}_{\mathbf{S}}\right) G_{\infty}\left(\hat{\mathbf{r}}, \mathbf{r}_{\mathbf{S}}\right)\right) d S\left(\mathbf{r}_{\mathbf{S}}\right) .
\end{aligned}
$$

Furthermore, like Eq. (3), Eqs. (7) and (8) are valid when $p_{t}$ is used instead of $p_{s}$ in the integral, provided that there are no sources inside $S$.

\section{Measurement method}

We assume that the pressure and normal particle velocity can be obtained on a surface $S$ enclosing the sample at $M$ discrete positions $\mathbf{r}_{j}(1 \leq j \leq M)$. Such measurements are possible with a sound intensity probe. ${ }^{27}$ Equation (7) can be discretized to obtain a matrix equation in the form

$$
\mathbf{f}_{\infty}=\mathbf{A p}+\mathbf{B} \mathbf{u}_{\mathbf{n}}
$$

where $\mathbf{p}$ and $\mathbf{u}_{\mathbf{n}}$ are vectors of size $M$ containing the measured pressures and normal particle velocities, respectively, and $\mathbf{f}_{\infty}$ is the estimated discrete far-field pattern in $N$ angular directions $\hat{\mathbf{r}}_{i}(1 \leq i \leq N)$. The elements of the matrices $\mathbf{A}$ and $\mathbf{B}$ are calculated from Eq. (7) and depend on the interpolation scheme chosen to discretize the integral (see Ref. 29, for instance). The choice of the interpolation is out of the scope of this paper.

Equation (9) makes it possible to estimate the far-field pattern from near-field measurements of the pressure and particle velocity. It is similar to the boundary element method (BEM), in which the scattered pressure is obtained from the sound field on the object's boundaries, based on Eq. (3). ${ }^{30}$ The difference is that in BEM, the sound field on the boundaries is calculated numerically, whereas the present method relies on measured sound field quantities. Furthermore, BEM does not directly yield the far-field pattern, which can still be approximated by calculating the scattered pressure far from the sample. Nevertheless, it could be possible to develop an asymptotic version of BEM that directly calculates the far-field pattern, based on the discretization of Eq. (7) instead of Eq. (3).

It is worth noting that Eq. (9) is a linear forward problem, which makes it robust to noise. Furthermore, similarly to Eq. (7), either the total or the scattered field can be used in the vectors $\mathbf{p}$ and $\mathbf{u}_{\mathbf{n}}$. Therefore, it is possible to estimate the far-field pattern without separating the incident and reflected fields (as in Refs. 26 and 15). Finally, the setup does not require large distances between the sample and measurement locations (as in Refs. 14 and 10).

\section{NUMERICAL RESULTS: STUDY OF FAR-FIELD PATTERNS}

Numerical simulations are performed using the twodimensional (2D) version of OpenBEM ${ }^{31}$ in order to analyze the features of the far-field pattern for different samples. A 2D simulation allows for faster calculations than full threedimensional (3D) models, and simplifies the analysis of the results as the far-field pattern then depends on only one angle. Finite-size effects are expected to be different from actual samples, but this is not problematic when studying the properties of the far-field pattern as a function. The geometries are discretized using six elements per minimum wavelength, as recommended in Ref. 30.

\section{A. Simulation setup}

Sketches of the studied samples are presented in Fig. 2. The three samples have a width $L=1.07 \mathrm{~m}$ and thickness $l=10 \mathrm{~cm}$. They have different upper boundaries,

- Sample A: flat and rigid, where specular reflection is expected to dominate;

- Sample B: flat and absorptive, where the upper surface represents a porous absorber of flow resistivity $10900 \mathrm{~N} \mathrm{~s}$ $\mathrm{m}^{-4}$ and thickness $10 \mathrm{~cm}$;

- Sample C: sinusoidal and rigid, with an amplitude $h=5.2 \mathrm{~cm}$ and spatial period $\Lambda=18.5 \mathrm{~cm}$, where we expect the energy to be redirected in specific directions at high frequencies. ${ }^{32}$

The side and lower boundaries are defined as rigid for the three samples. Note that the sinusoidal upper boundary makes sample $\mathrm{C}$ thicker than the two others, its total thickness being $20.4 \mathrm{~cm}$. Moreover, for sample B, the surface impedance of the upper boundary, which is needed for the 


\section{Sample A}

$l \uparrow$

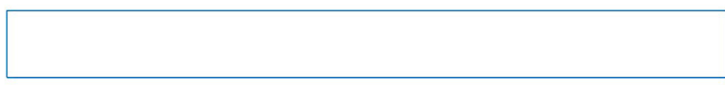

Sample B

$l$
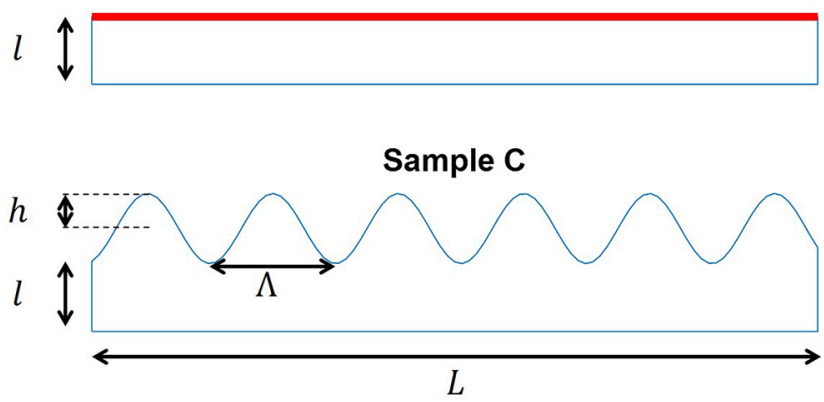

FIG. 2. (Color online) Tested sample geometries. (A) flat rigid surface, (B) flat absorptive surface, (C) sinusoidal rigid surface.

BEM simulation, is obtained in two steps. First, a characteristic impedance and a propagation constant are calculated with Miki's model. ${ }^{33}$ Then, these two parameters are used to infer the surface impedance following Eq. (3.39) of Ref. 34.

A position $\mathbf{r}$ is located using a polar coordinate system $(r, \theta)$, as shown in Fig. 3. The samples are placed in free-field and subjected to an incident plane wave. The angle of incidence of the plane wave $\phi_{0}$ is defined from the normal to the upper boundary, so that normal incidence corresponds to $0^{\circ}$. Under these conditions, the far-field pattern of a given sample only depends on the incidence angle and frequency. The tests are carried out at 500 and $2500 \mathrm{~Hz}$ for incidence angles of $0^{\circ}$ and $45^{\circ}$.

The BEM model makes it possible to calculate the pressure at any location with the same computational cost. Consequently, the scattered pressure is calculated on a large circle of radius $10^{6} \mathrm{~m}$, with an angular resolution of $0.5^{\circ}$. In $2 \mathrm{D}$, the radiating waves follow a cylindrical divergence, so Eq. (2) becomes

$$
p_{S}(\mathbf{r}) \underset{r \rightarrow+\infty}{=} \frac{e^{-j k r}}{\sqrt{r}}\left(f_{\infty}(\theta)+O\left(\frac{1}{r}\right)\right),
$$

and the far-field pattern is estimated by compensating for the radial dependence $e^{-j k r} / \sqrt{r}$. At such a large distance, the far-field pattern can be estimated with negligible error.

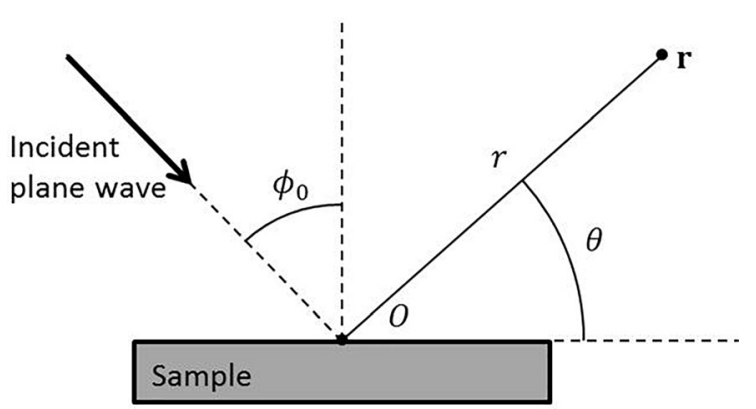

FIG. 3. Simulation setup and definition of the polar coordinate system.
For one given frequency and one incidence angle, the calculation of the far-field pattern takes approximately $1 \mathrm{~min}$ for a CPU speed of $1.6 \mathrm{GHz}$. The computation time is ascribed to the implementation of the BEM algorithm and, in particular, the calculation of the interpolation matrices.

\section{B. Results}

Figure 4 shows the amplitude of the far-field patterns for the three tested samples. Two key angular directions are indicated by dashed vertical lines. The specular direction $\theta_{0}=90^{\circ}-\phi_{0}$ corresponds to the direction of the reflected wave for an infinite flat surface. The forward direction $\theta_{f}=270^{\circ}+\phi_{0}$ is the direction of propagation of the incident plane wave. Additional dashed lines correspond to the angles at which the reflected field is redirected for an infinite sinusoidal surface of spatial period $\Lambda$, according to the formula ${ }^{32}$

$$
\cos \left(\theta_{n}\right)=\cos \left(\theta_{0}\right)+n \frac{\lambda}{\Lambda}
$$

where $\lambda$ is the acoustic wavelength. Note that $\theta_{0}$ is always a solution of Eq. (11) for $n=0$. In addition, multiple reflected directions only exist if the right-hand side of Eq. (11) is between -1 and 1 . For instance, at normal incidence, this implies that $\lambda<\Lambda$, or $f>c / \Lambda$. These additional lines, thus, only appear at $2500 \mathrm{~Hz}$ in Figs. 4(b) and 4(d).

In all cases, the far-field pattern amplitudes present lobes due to the finite size of the samples. The far-field pattern has a sinc-like behavior similar to the far-field Fraunhofer solution for flat rigid panels. ${ }^{12}$ Local maxima can be observed in the specular and forward directions. The specular lobe shows the existence of a reflection above the samples, whereas the forward one indicates a shadowing effect behind the samples. In Fig. 4(a), at normal incidence and $500 \mathrm{~Hz}$, samples $\mathrm{A}$ and $\mathrm{C}$ have similar far-field patterns. Indeed $\Lambda \ll \lambda$ so the sinusoidal upper boundary behaves like a flat surface. The specular and forward lobes have the same amplitude, which seems to be characteristic of totally reflective samples with no surface scattering. The far-field pattern of sample B resembles the two others, but it is attenuated in the directions above the sample $\left(\theta \leq 180^{\circ}\right)$ due to absorption by the upper surface. In Fig. 4(b), at $2500 \mathrm{~Hz}$, the lobes are narrower and the amplitude maxima are higher. As frequency increases, the far-field patterns converge toward the theoretical behavior of infinite surfaces under plane wave incidence, where the energy is reflected in discrete directions. The far-field pattern of sample A presents a specular and a forward main lobe, both having the same amplitude. Sample B shows a strong attenuation of the far-field pattern for angles below $180^{\circ}$, again due to absorption on its upper surface, whereas it behaves like the flat sample above $180^{\circ}$. For sample $\mathrm{C}$, additional peaks can be seen at $42^{\circ}$ and $138^{\circ}$, where a part of the reflected energy is redirected, as predicted by Eq. (11). Above $180^{\circ}$, its far-field pattern resembles the two others with small differences outside of the interval $\left[240^{\circ} ; 300^{\circ}\right]$, attributed to its larger thickness. At $45^{\circ}$ incidence for the three samples, the left side is more exposed to the incident field than at normal incidence, so it has more influence on the scattered field. In Fig. 4(c), at $500 \mathrm{~Hz}$, the 

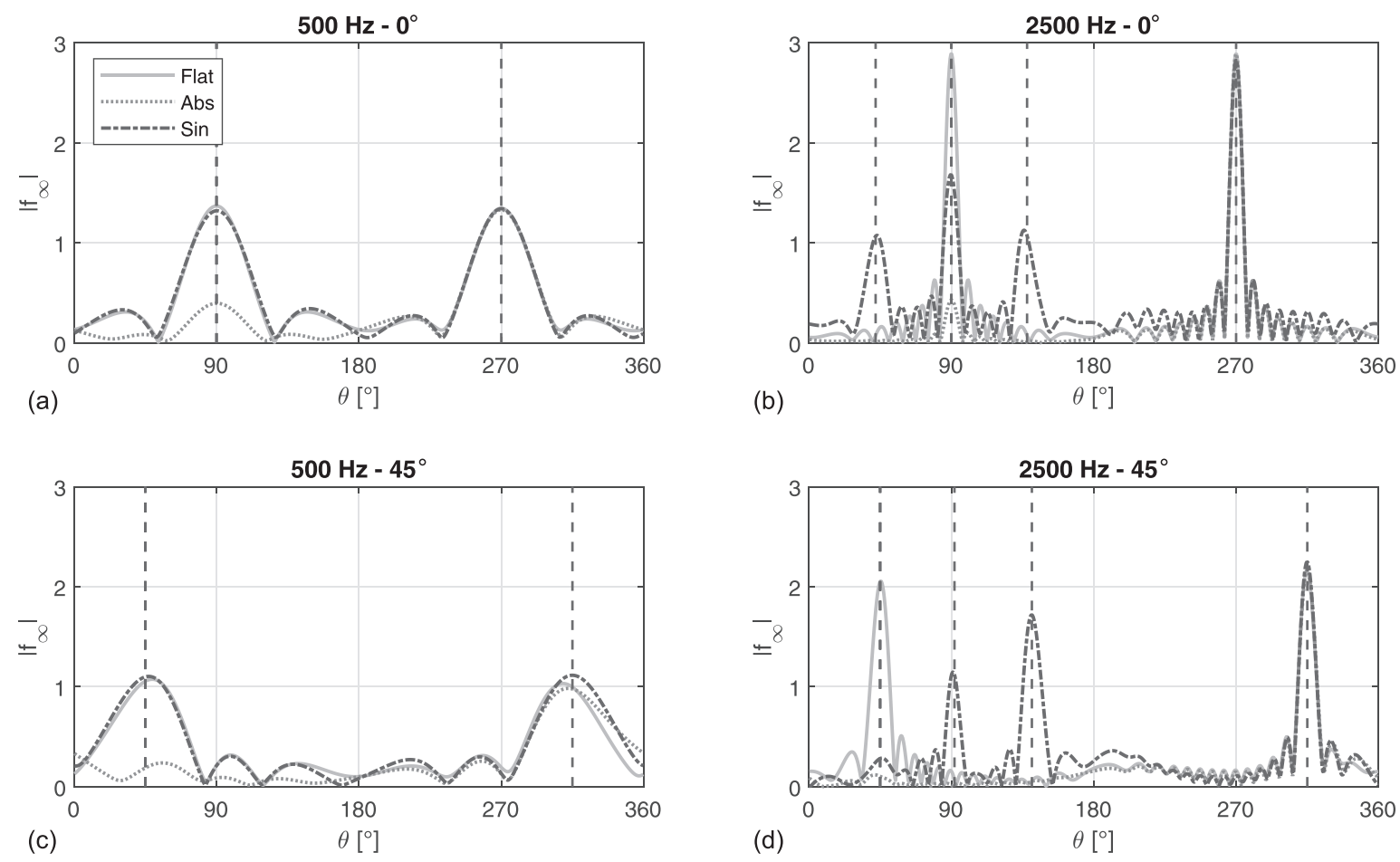

FIG. 4. Far-field pattern amplitudes of the three samples (-, flat sample A; $\cdots$, absorptive sample $\mathrm{B} ;-\cdot-$, sinusoidal sample $\mathrm{C}$ ) at $500 \mathrm{~Hz}$ and $2500 \mathrm{~Hz}$, incidence angles of $0^{\circ}$ and $45^{\circ}$. The vertical dashed lines indicate the specular direction $\theta_{0}$, the forward direction $\theta_{f}$, and the expected reflected directions $\theta_{n}$ for the sinusoidal surface.

observations made at normal incidence are still valid. In addition, the two main lobes overlap on the right side of the samples around $0^{\circ}$ and $360^{\circ}$, which increases the far-field pattern amplitude in this region. In Fig. 4(d), at $2500 \mathrm{~Hz}$, the samples show the same features as at normal incidence, such as strong lobes in the reflected directions. Furthermore, for all samples, the far-field pattern amplitude is higher than at normal incidence around $180^{\circ}$. This increase represents reflection by the left side of the samples, whose length is comparable to the wavelength $(\lambda=14 \mathrm{~cm})$. Sample $C$ behaves differently than the two other samples due to its larger thickness.

In order to illustrate the dependence of the far-field pattern on sample size, Fig. 5 shows the amplitude of the farfield pattern for samples similar to sample $\mathrm{A}$, with a flat rigid upper surface and different widths $(1 \mathrm{~m}, 2 \mathrm{~m}$, and $5 \mathrm{~m})$. The frequency is set to $500 \mathrm{~Hz}$ and the incidence angle is $0^{\circ}$. As the width increases, the far-field pattern converges toward a Dirac-like function with a peak in the specular direction $\left(90^{\circ}\right)$ and a peak in the forward direction $\left(270^{\circ}\right)$. Note that the effect is similar to what occurs at higher frequencies, as evidenced by Figs. 4(b) and 4(d). In conclusion, the oscillations observed in the far-field pattern are both due to frequency and sample size as expected.

In general, these examples show that the far-field pattern can be separated into two regions: the directions below $180^{\circ}$ represent the reflected field, while the ones above $180^{\circ}$ describe the shadowing effect of the sample. At high frequencies, this separation agrees well with the Kirchhoff approximation, where the scattered field is decomposed into a reflected component, dominating in backward directions, and a shadowing component prevalent in forward directions. ${ }^{35}$ The shadowing part depends on the shadow cross section, i.e., the maximum area covered by the sample in the direction perpendicular to the incident wave. Consequently, the forward part is similar for the three samples at high frequencies and small incidence angles. It should be noted that the angular separation between a reflected and shadowed component is only possible because the undisturbed field contains a single wave traveling in one direction, i.e., toward the upper surface of the sample. The reflected wave is then the only component traveling backwards. Under more complex undisturbed sound fields

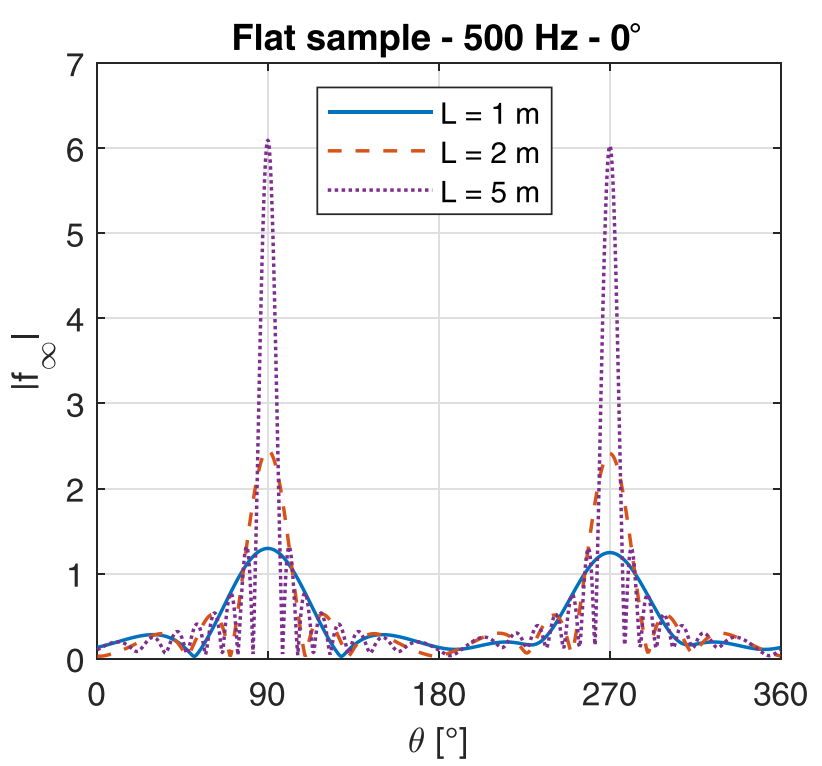

FIG. 5. (Color online) Far-field pattern amplitude for flat reflective samples of $1 \mathrm{~m}, 2 \mathrm{~m}$, and $5 \mathrm{~m}$ at $500 \mathrm{~Hz}$ under $0^{\circ}$ incidence. 
with several waves traveling in multiple directions, shadowing and reflection by the sample will be mixed in the resulting far-field pattern.

\section{Application: Calculation of architectural acoustics coefficients}

The backward part of the far-field pattern for $\theta \in\left[0^{\circ} ; 180^{\circ}\right]$ contains information on the properties of the upper surfaces such as absorption and surface scattering. The surface effects are particularly visible at high frequencies where the wavelength is much smaller than the sample width. Moreover, in free-field, the far-field pattern for $\theta \leq 180^{\circ}$ can be assimilated to the angular distribution of the reflected pressure, which is commonly used in the literature to estimate scattering by surfaces. ${ }^{10,36}$ Therefore, a potential application is to make use of the far-field pattern to infer already known coefficients, as suggested in a previous study. ${ }^{28}$ Simple examples of calculations are presented. However, the point of this study is not to develop a full methodology to obtain these coefficients, as the far-field pattern rather aims at the characterization of finite samples.

With regard to surface scattering, the derivation of the standardized diffusion coefficient is straightforward as it is based on the autocorrelation of the reflected pressure. ${ }^{7}$ Specifically, if the far-field pattern is known in $N$ discrete directions $\theta_{i}(i=1, \ldots, N)$ above the sample, the diffusion coefficient is calculated with

$$
\delta=\frac{\left(\sum_{i=1}^{N}\left|f_{\infty}\left(\theta_{i}\right)\right|^{2}\right)^{2}-\sum_{i=1}^{N}\left|f_{\infty}\left(\theta_{i}\right)\right|^{4}}{(N-1) \sum_{i=1}^{N}\left|f_{\infty}\left(\theta_{i}\right)\right|^{4}},
$$

assuming that the far-field pattern is sampled in uniform directions. ${ }^{3}$ It indicates the uniformity of the reflection. Figure 6 shows the calculated diffusion coefficient for the three samples at $0^{\circ}$ incidence. For the three samples, the diffusion coefficient is highest at low frequencies (about 0.6) where the far-field pattern is smoother [see Fig. 4(a)]. In this frequency range, scattering is mostly due to the finite size of the samples, which explains the similarity between the three curves, as the samples have the same width. Overall, samples $\mathrm{A}$ and $\mathrm{B}$ behave the same on the whole frequency range, which is sensible as both have a flat upper surface. As for sample $\mathrm{C}$, it redirects sound above $1.8 \mathrm{kHz}$ according to $\mathrm{Eq}$. (11), therefore, the diffusion coefficient increases again above $2 \mathrm{kHz}$.

Concerning the scattering coefficient, the samples are in free-field, therefore it is not possible to use the ISO definition, which requires a diffuse field. ${ }^{6}$ Moreover, the rotational definition introduced in Ref. 9 cannot be applied in two dimensions. Instead, the definition proposed by Mommertz, ${ }^{36}$ which is also based on the angular distribution of the reflected pressure, can be estimated with the far-field pattern, restricted to backward directions. The Mommertz coefficient is calculated as

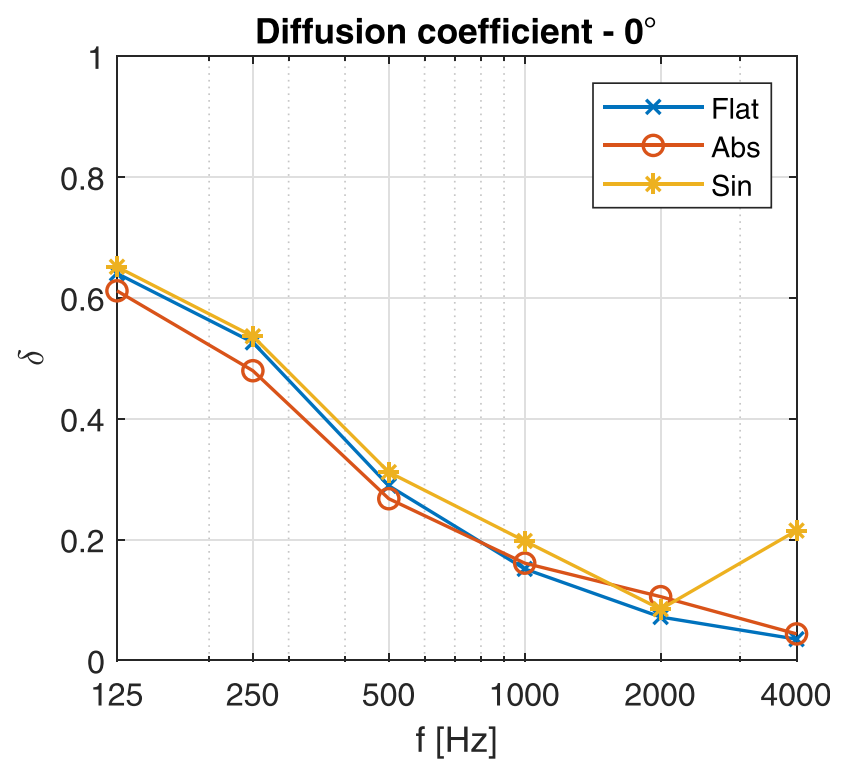

FIG. 6. (Color online) Estimated diffusion coefficient for the reflective sample A, the absorptive sample $\mathrm{B}$, and the sinusoidal sample $\mathrm{C}$ as a function of frequency under normal incidence.

$$
s_{M}=1-\frac{\left|\sum_{i=1}^{N} f_{\infty}\left(\theta_{i}\right) f_{\infty, \mathrm{ref}}^{*}\left(\theta_{i}\right)\right|^{2}}{\sum_{i=1}^{N}\left|f_{\infty}\left(\theta_{i}\right)\right|^{2} \sum_{i=1}^{N}\left|f_{\infty, \mathrm{ref}}\left(\theta_{i}\right)\right|^{2}},
$$

where $f_{\infty, \text { ref }}$ is the far-field pattern of a reference (typically a flat rigid sample of the same dimensions as the tested sample). This coefficient measures the dissimilarity of the reflection from the reference, so the latter should be representative of a specular reflection with no surface scattering. Sample A is thus chosen here as a reference. Figure 7 shows the Mommertz scattering coefficient for samples B and C. For the absorptive sample $\mathrm{B}$, the scattering coefficient is overall

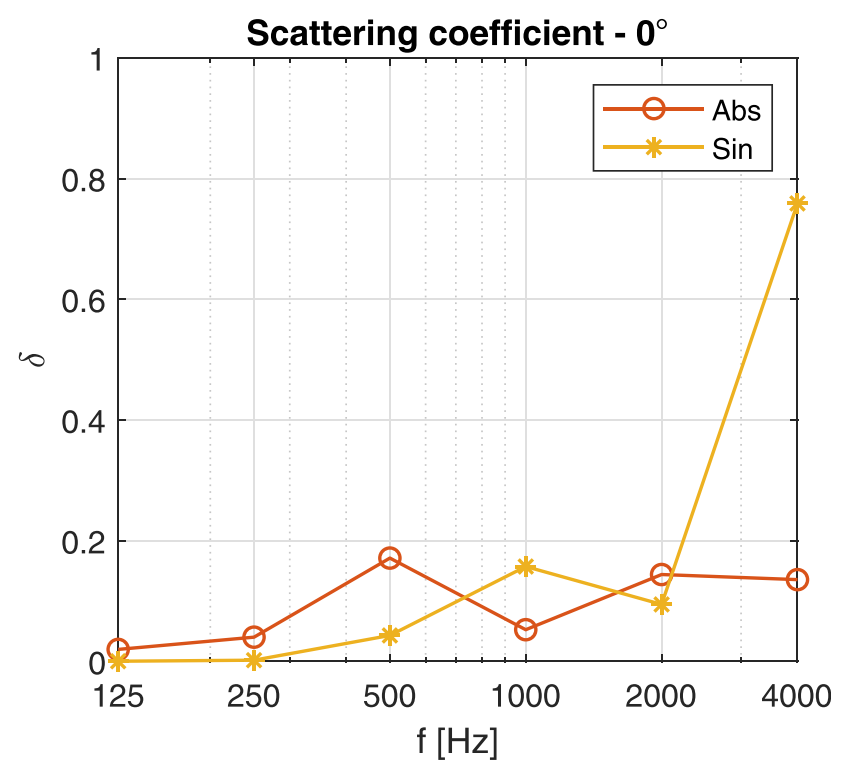

FIG. 7. (Color online) Estimated Mommertz scattering coefficient for the absorptive sample $\mathrm{B}$ and sinusoidal sample $\mathrm{C}$ as a function of frequency under normal incidence. Sample A is used as a reference in the calculation. 
below 0.2, as it has a flat upper surface. However, it is greater than zero because the far-field pattern of sample B differs from the far-field pattern of sample A due to absorption by the surface. This illustrates that the reference sample should, generally, have the same absorption properties as the tested sample, which might be difficult to achieve in practice. Finally, sample $\mathrm{C}$ is reflective, just as sample A, which is then a more valid choice of reference. For sample C, $s_{M}$ increases with frequency. At low frequencies, as discussed in Sec. III B, it behaves like a flat surface, so $s_{M}$ is close to 0 . Above $1.8 \mathrm{kHz}$, the reflection propagates in several directions, which yields significant differences with the reference far-field pattern of sample A. This explains the sharp increase of $s_{M}$ above $2 \mathrm{kHz}$.

In addition, Fig. 4 showed that absorption reduces the far-field pattern amplitude in the reflected directions. We can also assume that the forward lobe is representative of the incident field. Therefore, for flat samples, a reflection factor $R$ is calculated as the ratio between the specular peak and forward peak. An absorption coefficient can then be obtained as

$$
\alpha=1-|R|^{2}
$$

Figure 8 shows the estimated absorption coefficient for the two flat samples, reflective $\mathrm{A}$ and absorptive $\mathrm{B}$, following Eq. (14). Theoretical references are also shown: for sample A, $\alpha$ is expected to be 0 . For sample B, a theoretical reflection factor is calculated with Eq. (3.44) of Ref. 34 under an infinite sample assumption, and the corresponding absorption coefficient is calculated with Eq. (14). For both samples, good agreement is found above $1000 \mathrm{~Hz}$. At lower frequencies, deviations with the theoretical references are visible as the lobes are also influenced by the finiteness of the samples. In particular, for sample $\mathrm{A}$, the negative absorption

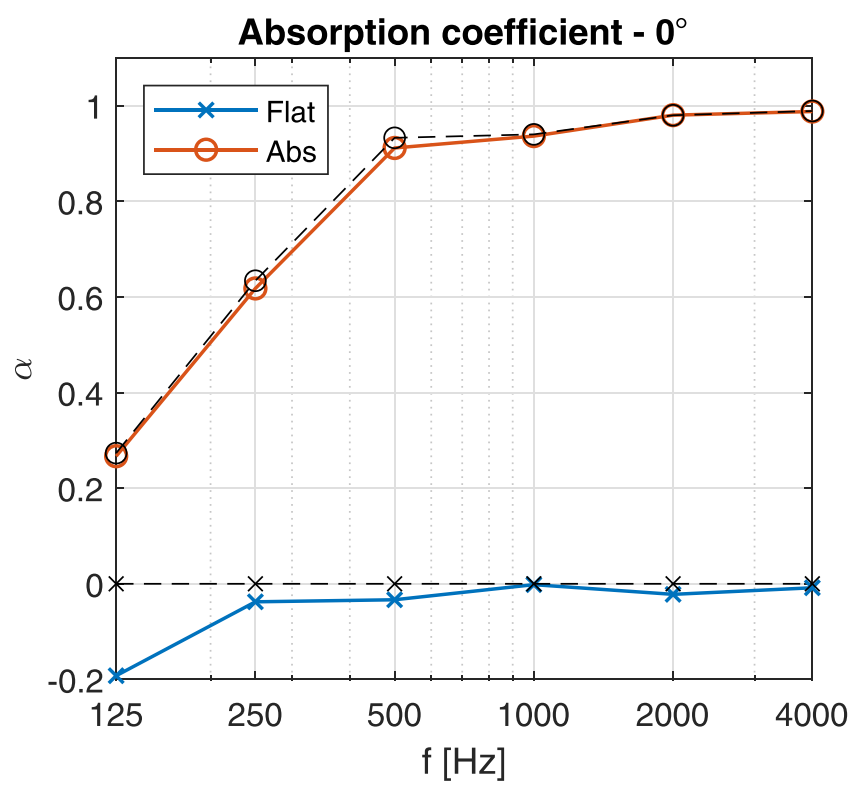

FIG. 8. (Color online) Absorption coefficient derived from the far-field pattern amplitude for the reflective sample A and absorptive sample B. The black lines indicate theoretical absorption coefficient values derived from a porous absorber model. coefficient indicates that the reflected lobe is actually stronger than the shadowing lobe. Overall, the estimation seems valid for $k L \geq 10$, where $L$ is the width of the sample (in the present example, $f \geq 500 \mathrm{~Hz}$ ).

In summary, the far-field pattern offers a complete description of scattering by the sample. It shows the directional properties of the scattered pressure and contains information on both absorption and scattering of sound by the upper surface. Moreover, the far-field pattern has a potential for estimating existing architectural acoustics coefficients. However, these traditional coefficients assume infinite surfaces, although they are necessarily measured with finite samples. Using the far-field pattern will not overcome this shortcoming as finite size effects are inherently included in the scattered field. Therefore, all the tested metrics are influenced by the sample size at low frequencies. The proposed coefficient derivations are only valid in cases where size effects are negligible. Typical examples are large surfaces, near normal incidence, and high frequencies. In other cases, the scattered field is a combination of effects due to the surface properties and the shape of the sample, which is entirely captured and described by the far-field pattern as a directivity measure.

\section{EXPERIMENTAL RESULTS}

Experimental tests were conducted in order to validate the feasibility of the measurement method presented in Sec. II C. The aim of these tests is to validate that far-field scattering information can be obtained from near-field measurements. A Microflown 3D sound intensity probe (Arnhem, Netherlands) was used to measure the pressure and particle velocity around the sample in a free-field environment.

\section{A. Setup}

The tests are carried out on two rectangular samples of dimensions $1.07 \mathrm{~m} \times 54 \mathrm{~cm} \times 10 \mathrm{~cm}$. The two samples are boxes made of wooden plates and filled with a porous absorber of flow resistivity $10900 \mathrm{~N} \mathrm{~s} \mathrm{~m}^{-4}$. The first sample has its front face covered with a flat wooden plate so that it is expected to be reflective, whereas the second sample has an open front face so it remains absorptive. These samples are similar to samples A and B in Sec. III so the same terminology is used in this section. The samples are suspended in a large anechoic room in the Technical University of Denmark (Kongens Lyngby, Denmark) of volume $1000 \mathrm{~m}^{3}$. A loudspeaker is placed in front of the samples at a distance of $3 \mathrm{~m}$. Stationary white noise is used as a source signal. A picture of the experimental setup is shown in Fig. 9.

A Scan\&Paint 3D system from Microflown (Arnhem, Netherlands) is used to manually scan the sound field around the sample. ${ }^{37}$ The system uses a 3D sound intensity probe comprising one pressure microphone and three orthogonal particle velocity transducers. The pressure and particle velocity signals are recorded with a multi-channel data acquisition system. As phased data are needed in Eq. (9), the electrical signal sent to the source is also recorded to serve as a phase reference. The position and orientation of the probe are tracked by a stereo camera (visible in Fig. 9). The 


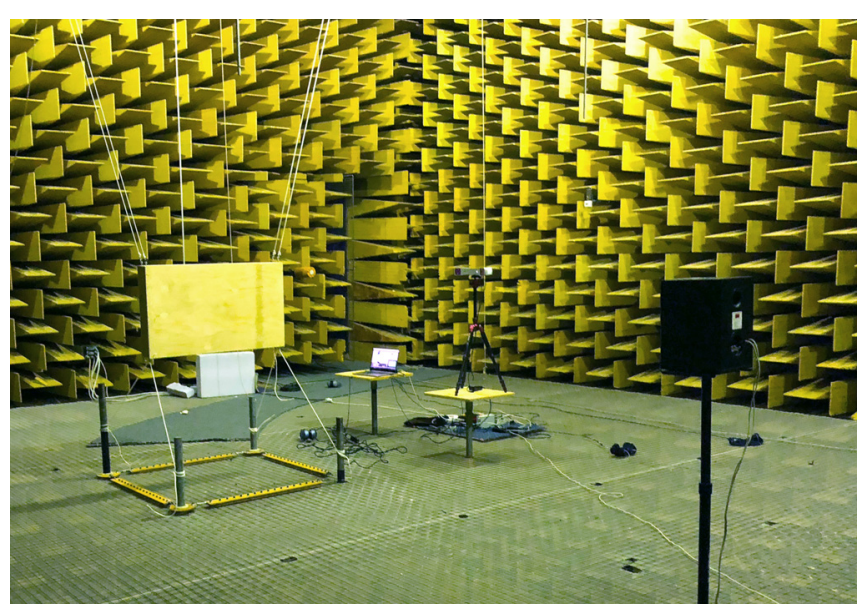

FIG. 9. (Color online) Experimental setup in the anechoic room. The sample is suspended in front of a loudspeaker. The camera and data acquisition system of the Scan\&Paint 3D system (Microflown, Arnhem, Netherlands) are also visible.

space is discretized into cubic cells of dimensions $2 \times 2$ $\times 2 \mathrm{~cm}^{3}$. A fast Fourier transform (FFT) is applied on each cell where the probe has been detected by the camera. In this experiment, the signals' sampling frequency is set to 24 $\mathrm{kHz}$, and 2048 samples are used per time window for the FFT. The scanning takes about $15 \mathrm{~min}$ and requires two different tracking camera locations to obtain a closed surface around the samples.

The resulting cloud of measurement points depends on the scanning process. Following Eq. (7), a surface enclosing the sample must be defined from the measured points. The surface must be relatively smooth and contain as many points as possible in order to obtain a good estimate of the far-field pattern. The MATLAB function alphashape, which produces a polygon enclosing the cloud of points, is used to select the points with an alpha radius of $12 \mathrm{~cm}$. The required particle velocity term in Eq. (7) is obtained by projecting the velocity vector on the normal to the obtained surface.
In this study, in order to simplify the implementation, the far-field pattern is calculated using the 3D BEM code OpenBEM. ${ }^{31}$ The scattered pressure is calculated at a distance of $10^{6} \mathrm{~m}$ from the origin, and the radial dependence is compensated for following Eq. (2). The far-field directions are sampled on a sphere of $61 \times 61$ points. With about 2300 near-field points and 3721 far-field directions, the calculation of the far-field pattern takes 8:30 min for each frequency. As in Sec. III, the computation speed depends on the implementation of the BEM code.

\section{B. Results}

Due to the relatively large size of the samples, the nearfield scanning procedure takes about 15 min per sample and leads to a rather coarse mesh of data points. The resulting measurement points that define an enclosing surface have an average distance of $6 \mathrm{~cm}$, which generates aliasing effects above $1 \mathrm{kHz}$. Therefore, the present results are only valid in the low- to mid-frequency range. A higher spatial resolution can be obtained by scanning the volume for a longer time.

Figure 10 shows estimated directivity plots for the flat sample A and absorptive sample B under normal incidence at 500 and $800 \mathrm{~Hz}$, respectively. The direction of propagation of the incident wave is from left to right as indicated by the arrow. The obtained directivity plots are suitable for a qualitative analysis of scattering. In Fig. 10(a), at $500 \mathrm{~Hz}$, sample A shows mainly a forward and backward lobe with much smaller side lobes, which result from the finiteness of the sample. The backward lobe has a smaller amplitude, which indicates that the surface of sample A is partially absorptive. Indeed, the sample is made of plywood and not entirely rigid at low frequencies. In Fig. 10(b), at $800 \mathrm{~Hz}$, the far-field pattern presents stronger and narrower side lobes. The backward and forward lobes are of more comparable size than at $500 \mathrm{~Hz}$, showing that the surface is more reflective at this frequency. For the absorptive sample B, the far- (a)

Sample A - $500 \mathrm{~Hz}$

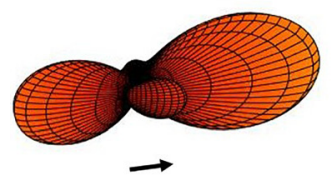

(c)

Sample B $-500 \mathrm{~Hz}$ (b)

Sample A $-800 \mathrm{~Hz}$

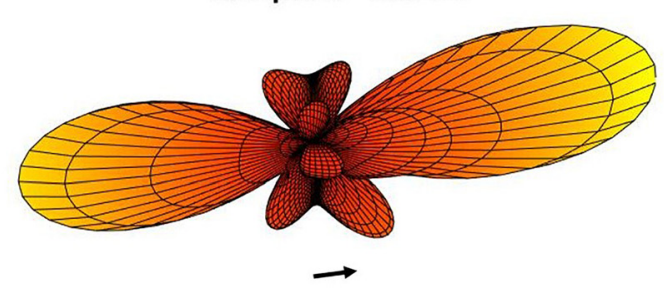

(d)

Sample B $-800 \mathrm{~Hz}$

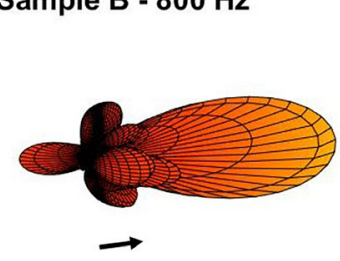

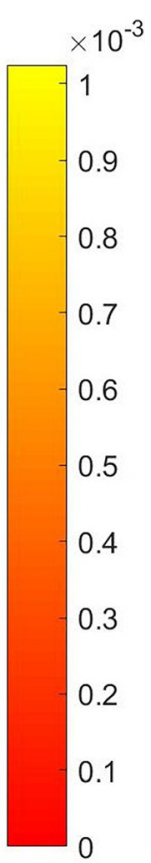

FIG. 10. (Color online) Far-field pattern amplitudes obtained experimentally for the reflective sample A and absorptive sample B at $500 \mathrm{~Hz}$ and at $800 \mathrm{~Hz}$, respectively. The arrows indicate the direction of propagation of the incident wave.

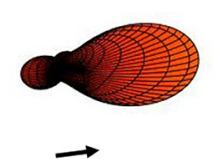


field pattern at $500 \mathrm{~Hz}$ in Fig. 10 (c) also shows two main lobes. The backward lobe has a much smaller amplitude than the forward lobe, which is due to absorption by the porous surface of the sample. Note that the forward lobe is smaller than for sample A, which is presumably due to transmission of sound through the sample. In Fig. $10(\mathrm{~d})$, at $800 \mathrm{~Hz}$, the lobes are again narrower and stronger with the presence of side lobes due to the sample's finiteness. Absorption by the surface is also clearly shown by the smaller amplitude of the backward lobe. Figure 10, thus, shows that the far-field pattern fully captures the scattering behavior of the sample in 3D.

In order to validate the experimental results and complement them with a quantitative analysis, the far-field patterns of samples A and B are restricted to the central azimuth plane and compared with the 2D BEM results from Sec. III. As the power of the source is a priori unknown, the far-field patterns are normalized by their maximal amplitude. Figure 11 shows the comparison for the two samples at $500 \mathrm{~Hz}$ and $800 \mathrm{~Hz}$ under normal incidence. It should be noted that the 3D experimental results are compared with a 2D model, which explains deviations, especially in the lower side lobes. In Fig. 11(a), for sample A at $500 \mathrm{~Hz}$, the location of the reflected and forward lobes agrees with the numerical results. The slight offset between the numerical and experimental results in the main lobe directions is attributed to a small misalignment of the sample and source in the experiment. The reflected lobe has a lower amplitude than the forward lobe, as seen in Fig. 10(a). In Fig. 11(b), at $800 \mathrm{~Hz}$, the two curves are similar not only around the main lobes, but also around side lobes such as at $56^{\circ}, 125^{\circ}, 236^{\circ}$, and $305^{\circ}$. In particular, the amplitudes of the forward and reflected lobes correspond quite well to the ones in BEM. In Fig. 11(c), sample B shows good agreement between the experimental and numerical results at $500 \mathrm{~Hz}$. The relative amplitude difference between the reflected lobe and forward lobe is similar for both curves, which indicates similar absorption properties between the sample and numerical model. In Fig. 11(d), at $800 \mathrm{~Hz}$, good agreement is found for the forward and reflected lobes, and as for sample A, some of the side lobes are also recovered. These results show that the near-field measurement method makes it possible to capture the main characteristics of the far-field pattern. However, the numerical and experimental setups are not entirely identical. For example, in the experiment, the incident field is not a plane wave, and the sample is not in perfect free-field. In addition, the samples may have different acoustical properties than their numerical models. For instance, the wooden box that is used for sample $\mathrm{A}$ is not perfectly rigid.

\section{DISCUSSION}

The far-field pattern is a suitable quantity to characterize scattering by objects as it is exact, unique, and offers a full $3 \mathrm{D}$ description of the scattered field. It can be applied to any object such as acoustic elements (absorbers, diffusers) or furniture. The object under characterization must, however, be of finite size, which makes the far-field pattern a sizedependent function. Furthermore, the far-field pattern depends on the undisturbed field. In this study, free-field and plane wave incidence have been assumed in order to simplify this dependence to only one parameter, namely the incidence angle. The scattered sound field behaves differently under other wave fronts, which has been shown, for example, for a porous layer backed by a hard surface under an incident spherical wave. ${ }^{38}$ The far-field pattern of a given object is, therefore, different if it is placed in another environment or subject to another incident field. (a)

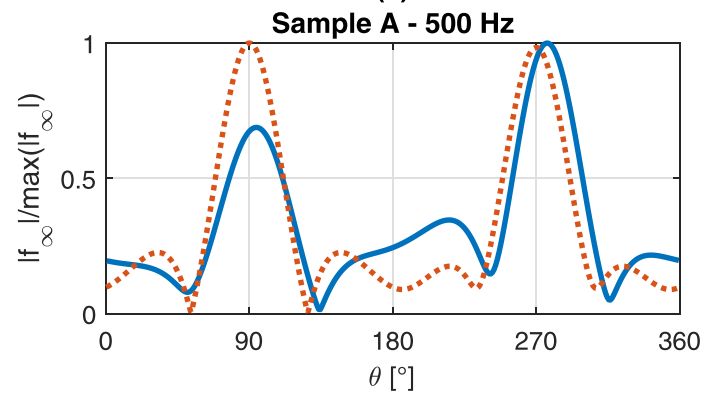

(c)

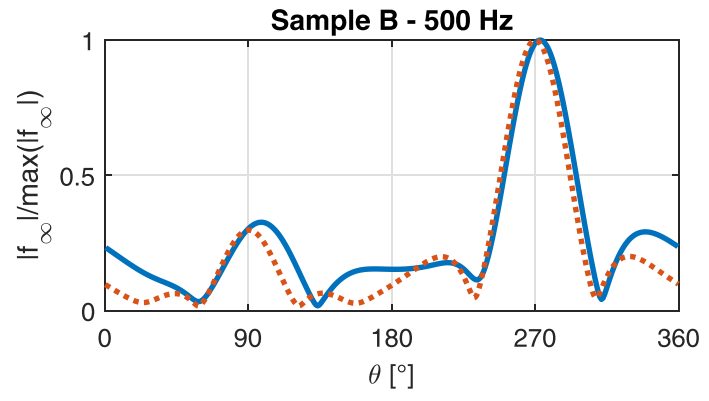

(b)

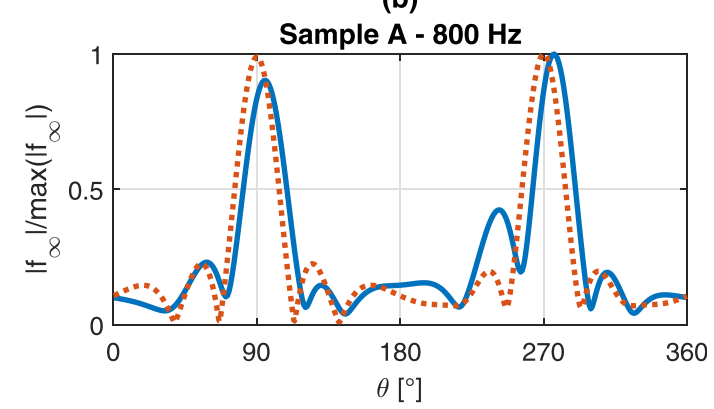

(d)

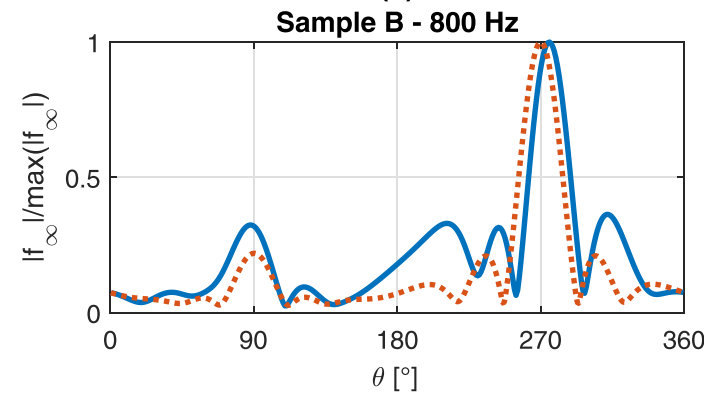

FIG. 11. (Color online) Comparison of the experimental far-field patterns ( - ) and the numerical results $(\cdots)$ over a horizontal circle for the reflective sample A and the absorbing sample B, respectively, at $500 \mathrm{~Hz}$ and at $800 \mathrm{~Hz}$, and normal incidence. 
The proposed measurement method relies on new sensing principles (near-field pressure and particle velocity measurement) and an exact formulation of the scattering problem, using the HIE. Fundamentally, scattering is described in the far-field where the sound field behaves regularly, and based on near-field information, which can be measured at attainable distances from the sample. The mathematical propagation from the near-field to the far-field is a linear forward operation, which smooths data and limits the sensitivity to noise. The proposed scanning procedure is carried out on an arbitrary closed surface with one single probe. This relaxes geometrical constraints introduced in the existing near-field array methods to study scattering (e.g., planar $^{15}$ or spherical $\left.{ }^{26}\right)$. Moreover, the use of a closed measurement surface means that neither truncation of the underlying equations ${ }^{15}$ nor subtraction of the incident field $^{15,26}$ are required. One challenge is the quality of the measured input data. The estimation of the far-field pattern relies on phased data, and especially on the phase difference between pressure and particle velocity. Therefore, the method is sensitive to the phase mismatch between the measurement channels of the probe. Moreover, the use of the HIE is generally a low-frequency approach. Although it is, in theory, applicable in any frequency range, higher frequencies require a finer spatial resolution and, hence, an increasing amount of near-field data. In BEM methods, a spacing of $\lambda / 6$ is typically recommended. ${ }^{30}$

The current experimental validation proves the feasibility of the measurement principle. However, several improvements are needed to fully characterize scattering, especially by rough surfaces. First, the measurements were carried out in the free-field with a single incident wave. A total characterization would thus require repeating the measurement for other incidence angles. This is a typical drawback of freefield methods to measure angle-dependent properties. ${ }^{10}$ On the other hand, placing multiple receivers in the far-field to describe the directional properties of the scattered field (as in Refs. 10 and 14) is no longer needed. Furthermore, in the presented experimental setup, the samples are hung freely in the air so that they are the only source of scattering. This situation rarely occurs in practice and tends to amplify edge diffraction. More realistic scenarios, such as a diffuser placed on a wall, should therefore be considered. In that case, however, the undisturbed field also contains reflection by the wall, which travels away from the sample's surface. The scattered field and subsequent far-field pattern then also include interaction with this reflection. ${ }^{28}$ A possible solution to treat more complex sound fields is to use a different Green's function in Eq. (8), which describes a more appropriate wave propagation. Finally, rough surface scattering is not visible in the present experimental results as it typically occurs at higher frequencies. This can be overcome with a more thorough and longer scanning of the volume. Nevertheless, it is also possible to optimize the processing of the measured data. Indeed, many measurement points were discarded in order to define an enclosing surface (typically about $60 \%$ of them). The upper frequency limit could be increased by including this measured information in the calculation, for instance, with near-field holography techniques. $^{39}$

Finally, the far-field pattern and its measurement show potential applications in architectural acoustics. The absorptive and scattering properties of surfaces are included in this directivity function, so techniques can be implemented to derive traditional measures such as absorption and scattering coefficients. In fact, the far-field pattern is conceptually close to the directional distribution of the reflected pressure, which has been extensively used in the literature for characterizing surface scattering. ${ }^{0,15,26,36}$ Such approaches are directly applicable by substituting the reflected pressure with the farfield pattern. Nevertheless, the far-field pattern and reflected pressure are size dependent, so the estimation is only valid when surface reflection dominates over finite-size effects. This is the case, for example, for large surfaces $(k L \geq 10)$ and small incidence angles. More generally, scattering, as defined in this study, contains a combination of effects due to shape and boundary conditions so it cannot simply be understood as a surface property. Instead, it is possible to characterize a given sample with its far-field pattern, including absorption, surface scattering, and finiteness simultaneously. The far-field pattern could then serve as an input to numerical simulations in a similar way as the BRDF. ${ }^{23}$ The far-field pattern differs from the BRDF as it describes an entire object-not a surface. However, it can be argued that such a directivity function represents an overly large amount of information for inclusion in numerical models. ${ }^{14,15}$

\section{CONCLUSION}

This study proposes a framework to characterize scattering by finite samples. Scattering is defined as the disturbance of the sound field by the sample due to its shape and boundary conditions. It is described with a complex directivity function called the far-field pattern.

The far-field pattern provides a full 3D directional representation of the scattered field, and it includes absorption, rough surface scattering, and finite-size effects simultaneously. Therefore, the far-field pattern can potentially be used in architectural acoustics to describe the effect of any given object on the sound field, such as furniture, absorbers, or diffusers. However, it is by definition a size-dependent function.

A near-field measurement method with a 3D sound intensity probe was proposed and validated experimentally, based on the measurement of pressure and particle velocity. The methodology offers a robust estimation of the far-field pattern and a simple scanning measurement procedure. Although the methodology is also valid at higher frequencies, a long measurement duration is required to study frequencies above 1 $\mathrm{kHz}$, and hence to capture rough surface scattering effects. The method has been developed in free-field, but it can be extended to more complex environments such as actual rooms.

\section{ACKNOWLEDGMENTS}

The authors would like to thank Peter Svensson for fruitful discussions and Henrik Hvidberg for his help in the experimental setup. 
${ }^{1}$ L. Savioja and U. P. Svensson, "Overview of geometrical room acoustic modeling techniques,” J. Acoust. Soc. Am. 138(2), 708-730 (2015).

${ }^{2}$ E. Brandão, A. Lenzi, and S. Paul, "A review of the in situ impedance and sound absorption measurement techniques," Acta Acust. Acust. 101(3), 443-463 (2015).

${ }^{3}$ T. J. Cox, B. I. L. Dalenback, P. D’Antonio, J. J. Embrechts, J. Y. Jeon, E. Mommertz, and M. Vorländer, "A tutorial on scattering and diffusion coefficients for room acoustic surfaces," Acta Acust. Acust. 92, 1-15 (2006).

${ }^{4}$ ISO 354-2003: Acoustics-Measurement of Sound Absorption in a Reverberation Room (International Organization for Standardization, Geneva, Switzerland, 2003).

5 ISO 10534-2-1998: Acoustics—Determination of sound absorption coeffcient and impedance in impedance tubes (International Organization for Standardization, Geneva, Switzerland, 1998).

${ }^{6}$ ISO 17497-1-2004: Acoustics-Sound-Scattering Properties of Surfaces. Part 1: Measurement of the Random-Incidence Scattering Coefficient in a Reverberation Room (International Organization for Standardization, Geneva, Switzerland, 2004).

${ }^{7}$ ISO 17497-2-2012: Acoustics-Sound-Scattering Properties of Surfaces. Part 2: Measurement of the Directional Diffusion Coefficient in a Free Field (International Organization for Standardization, Geneva, Switzerland, 2012).

${ }^{8}$ H. Kuttruff, "Reflection and scattering," in Room Acoustics, 6th ed. (CRC Press, Boca Raton, FL, 2016), Chap. 2, pp. 35-66.

${ }^{9} \mathrm{M}$. Vorländer and E. Mommertz, "Definition and measurement of randomincidence scattering coefficients," Appl. Acoust. 60, 187-199 (2000).

${ }^{10}$ T. J. Hargreaves, T. J. Cox, Y. W. Lam, and P. D'Antonio, "Surface diffusion coefficients for room acoustics: Free-field measures.," J. Acoust. Soc. Am. 108(4), 1710-1720 (2000).

${ }^{11}$ J. H. Rindel, "Attenuation of sound reflections due to diffraction," in Proc. Nordic Acoustical Meeting, Aalborg (January, 1986), pp. 257-260.

${ }^{12}$ T. J. Cox, "Evaluation of methods for predicting the scattering from simple rigid panels," Appl. Acoust. 40(2), 123-140 (1993).

${ }^{13}$ E. Brandão, A. Lenzi, and J. Cordioli, "Estimation and minimization of errors caused by sample size effect in the measurement of the normal absorption coefficient of a locally reactive surface," Appl. Acoust. 73, 543-556 (2012).

${ }^{14} \mathrm{P}$. D'Antonio and J. Konnert, "The directional scattering coefficient: Experimental determination," J. Audio Eng. Soc. 40(12), 997-1017 (1992).

${ }^{15}$ M. Kleiner, H. Gustafsson, and J. Backman, "Measurement of directional scattering coefficients using near-field acoustic holography and spatial transformation of sound fields," J. Audio Eng. Soc. 45(5), 331-346 (1997).

${ }^{16} \mathrm{~K}$. Kimura and K. Yamamoto, "The required sample size in measuring oblique incidence absorption coefficient experimental study," Appl. Acoust. 63(5), 567-578 (2002).

${ }^{17}$ S.-1. Thomasson, "Theory and experiments on the sound absorption as a function of the area," TRITA-TAK-8201, Stockholm (1982).

${ }^{18}$ M. H. A. Gomes, M. Vorländer, and S. N. Y. Gerges, "Aspects of the sample geometry in the measurement of the random-incidence scattering coefficient," in Forum Acusticum, Sevilla (2002).

${ }^{19}$ S.-1. Thomasson, "On the absorption coefficient," Acustica 44, 265-273 (1980).
${ }^{20}$ D. Colton and R. Kress, Inverse Acoustic and Electromagnetic Scattering Theory (Springer, New York, 2013), pp. 13-71.

${ }^{21}$ G. Kriegsmann, A. Norris, and E. Reiss, "An optical theorem for acoustic scattering by baffled flexible surfaces," J. Sound. Vib. 99, 301-307 (1985).

${ }^{22}$ A. Levitas and M. Lax, "Scattering and absorption by an acoustic strip," J. Acoust. Soc. Am. 23, 316-322 (1951).

${ }^{23}$ S. Siltanen, T. Lokki, S. Kiminki, and L. Savioja, "The room acoustic rendering equation," J. Acoust. Soc. Am. 122(3), 1624-1635 (2007).

${ }^{24}$ T. J. Cox, "Prediction and evaluation of the scattering from quadratic residue diffusers," J. Acoust. Soc. Am. 95(1), 297-305 (1994).

${ }^{25} \mathrm{E}$. I. Thorsos, "The validity of the Kirchhoff approximation for rough surface scattering using a Gaussian roughness spectrum," J. Acoust. Soc. Am. 83(1), 78-92 (1988).

${ }^{26} \mathrm{M}$. Müller-Trapet, "Measurement of surface reflection properties: Concepts and uncertainties," Ph.D. thesis, RWTH Aachen University, 2015.

${ }^{27}$ D. Yntema, J. van Honschoten, H.-E. de Bree, R. Wiegerink, and M. Elwenspoek, "A three dimensional microflown," in 19th IEEE International Conference on Micro Electro Mechanical Systems, Istanbul, Turkey (January 2006), pp. 654-657.

${ }^{28}$ A. Richard, E. Fernandez-Grande, J. Brunskog, and C.-h. Jeong, "Characterization of acoustic scattering from objects via near-field measurements," in Proceedings of Euronoise, Crete, Greece (May 2018), pp. $2195-2202$.

${ }^{29}$ S. Marburg and S. Schneider, "Influence of element types on numeric error for acoustic boundary elements," J. Comput. Acoust. 11(3), 363-386 (2003).

${ }^{30} \mathrm{~N}$. Atalla and F. Sgard, Finite Element and Boundary Methods in Structural Acoustics and Vibration (CRC Press, Boca Raton, FL), pp. 233-273.

${ }^{31}$ V. Cutanda Henríquez and P. M. Juhl, "OpenBEM-An open source boundary element method software in acoustics," in Proceedings of Internoise, Lisbon, Portugal (June 2010).

${ }^{32}$ R. L. Holford, "Scattering of sound waves at a periodic, pressure-release: An exact solution," J. Acoust. Soc. Am. 70, 1116-1128 (1981).

${ }^{33} \mathrm{Y}$. Miki, "Acoustical properties of porous materials-Modifications of Delany-Bazley models," J. Acoust. Soc. Jpn. 11(1), 19-24 (1990).

${ }^{34}$ J. F. Allard and N. Atalla, "Acoustic impedance at oblique incidence in fluids. Substitution of a fluid layer for a porous layer," in Propagation of Sound in Porous Media: Modelling Sound Absorbing Materials, 2nd ed. (Wiley, Chichester, UK, 2009), Chap. 3, pp. 29-43.

${ }^{35}$ P. Y. Ufimtsev, Fundamentals of the Physical Theory of Diffraction (Wiley, Hoboken, NJ), Chaps. 1 and 2.

${ }^{36} \mathrm{E}$. Mommertz, "Determination of scattering coefficients from the reflection directivity of architectural surfaces," Appl. Acoust. 60, 201-203 (2000).

${ }^{37}$ D. Fernandez Comesaña, S. Steltenpool, M. Korbasiewicz, and E. Tijs, "Direct acoustic vector field mapping: New scanning tools for measuring 3D sound intensity in 3D space," in Proceedings of Euronoise, Maastricht, the Netherlands (June 2015), pp. 891-895.

${ }^{38}$ R. Dragonetti, M. Napolitano, and R. A. Romano, "A study on the energy and the reflection angle of the sound reflected by a porous material," J. Acoust. Soc. Am. 145(1), 489-500 (2019).

${ }^{39}$ Z.-W. Luo, D. Fernandez Comesaña, C.-J. Zheng, and C.-X. Bi, "Nearfield acoustic holography with three-dimensional scanning measurements," J. Sound. Vib. 439, 43-55 (2019). 